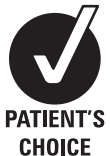

CHOICE

\title{
Freezing of gait in Parkinson's disease: a perceptual cause for a motor impairment?
}

\author{
$0 \mathrm{~J}$ Almeida, C A Lebold
}

Movement Disorders Research and Rehabilitation Centre, Wilfrid Laurier University, Waterloo, Ontario, Canada

\section{Correspondence to}

Dr Quincy J Almeida, Associate Professor and Director, Sun Life Financial Movement Disorders Research \& Rehabilitation Centre, Wilfrid Laurier University, 75 University Avenue West, Waterloo, Ontario, Canada N2L 3C5; qalmeida@ wlu.ca

Received 13 August 2008 Revised 24 July 2009 Accepted 14 August 2009

\section{ABSTRACT}

While freezing of gait (FOG) is typically considered a motor impairment, the fact that it occurs more frequently in confined spaces suggests that perception of space might contribute to FOG. The present study evaluated how doorway size influenced characteristics of gait that might be indicative of freezing. Changes in spatiotemporal aspects of gait were evaluated while walking through three different-sized doorways (narrow $(0.675 \mathrm{~m}$ wide $\times 2.1 \mathrm{~m}$ high), normal $(0.9 \mathrm{~m}$ wide $\times$ $2.1 \mathrm{~m}$ high) and wide $(1.8 \mathrm{~m}$ wide $\times 2.1 \mathrm{~m}$ high) $)$ in three separate groups: 15 individuals with Parkinson's disease confirmed to be experiencing FOG at the time of test; 16 non-FOG individuals with Parkinson's disease and 16 healthy age-matched control participants. Results for step length indicated that the FOG group was most affected by the narrow doorway and was the only group whose step length was dependent on upcoming doorway size as indicated by a significant interaction of group by condition $(F(4,88)=2.73, p<0.034)$. Importantly, the FOG group also displayed increased within-trial variability of step length and step time, which was exaggerated as doorway size decreased $(F(4,88)=$ 2.99, $\mathrm{p}<0.023$ ). A significant interaction between group and condition for base of support measures indicated that the non-FOG participants were also affected by doorway size (similar to Parkinson's disease FOG) but only in the narrow doorway condition. These results support the notion that some occurrences of freezing may be the result of an underlying perceptual mechanism that interferes with online movement planning.

Although not present in all patients, freezing is perhaps the most debilitating symptom of Parkinson's disease as it may lead to falls, a decrease in quality of life and loss of independence. ${ }^{1-3}$ Nearly one third of Parkinson's disease patients experience some type of freezing episode. ${ }^{4}$ Freezing of gait (FOG) is characterised by a sudden inability to initiate or continue walking, especially while turning, in stressful time-constrained situations and upon entrance into and through confined spaces such as doorways. ${ }^{5-9}$ Increased stride-tostride variability has recently been identified before FOG (compared with Parkinson's disease patients without FOG) during a $20 \mathrm{~m}$ "stand up and go" walking task. ${ }^{10}$ Hausdorff et al demonstrated that the ability to regulate stride-to-stride timing during gait is severely impaired in FOG patients compared with other individuals with Parkinson's disease. Hence, analysis of stride-to-stride variability is a useful method of identifying characteristics of gait that are closely linked to freezing. Parkinson's disease patients with FOG also display altered timing and, specifically, premature muscle activation and termination patterns before a freezing episode, leading to an abnormally long stance phase. ${ }^{11-14}$ Thus, the evidence for a central timing deficit in Parkinson's disease ${ }^{15} 16$ is growing.

While freezing has been argued to be the result of a motor block, ${ }^{4}$ recent evidence has suggested other possible factors that may contribute. In their more recent work, Giladi et al argue that FOG must have a different pathophysiology than typical motor symptom, since other motor issues are positively influenced by dopaminergic medication, while freezing remains unresponsive. ${ }^{17}$ While Okuma et $a l{ }^{6}$ point out that FOG can be sensitive to medication, most research has supported the notion that FOG is dopa-resistant. ${ }^{9}{ }^{18}$ FOG has also been linked to secondary issues that are common in Parkinson's disease, such as anxiety, depression, stress and panic. $^{17}{ }^{19-21}$

Perception may be the most important alternate mechanisms to consider. While perceptual influences associated with freezing are rarely considered, Parkinson's disease patients are profoundly influenced by awareness of their body (relative to environment). ${ }^{22}$ In spatial perception tasks, individuals with Parkinson's disease require a greater number of saccades to create accurate spatial representations. ${ }^{23}$ While this study concluded that spatial perception is comparable in healthy and Parkinson's disease participants, Parkinson's disease with FOG may be uniquely influenced by space perception.

Importantly, perceptual judgement deficits have been recently identified as a contributing factor to motor impairment in Parkinson's disease. ${ }^{24}$ Collectively, research suggests that key differences exist in the perceptual processing capabilities of Parkinson's disease patients with FOG specifically, highlighting the potential for a relationship to exist between perception and FOG. ${ }^{25}$

Currently, research investigating why freezing occurs while travelling through confined spaces remains incomplete. One possibility is that impaired integration of vision with spatial memory prevents patients with FOG from adapting to confined spaces. In a qualitative study, Parkinson's disease patients reported feeling too large to pass through small spaces, even though they were aware that doorways are designed for human size. ${ }^{26}$ Lee et al found that individuals with Parkinson's disease who responded yes to the question "Have you ever had problems walking through narrow spaces?" were also likely to be subject to difficulties with freezing. ${ }^{26}$ Yet, to our knowledge, there is no research quantifying whether an alteration in gait occurs in anticipation of a confined space. By examining changes to gait before a confined space, 
it may be possible to determine whether a perceptual mechanism might contribute to or trigger FOG.

Thus, the aim of this experiment was to evaluate the effect of doorway size on gait before reaching the doorway in two groups of individuals with Parkinson's disease: (1) those who experience freezing of gait (Parkinson's disease FOG) and (2) those who experience gait abnormalities but are absent of FOG (Parkinson's disease Non-FOG).

\section{SUBJECTS AND METHODS Participants}

The study involved 31 participants with Parkinson's disease (15i confirmed to be experiencing FOG at the time of test, 16 absent of FOG) and 16 healthy, age-matched control participants (no significant differences for age, height or symptom severity between groups; for full details of participant characteristics, see table 1) recruited from a database at the Movement Disorders Research and Rehabilitation Centre at Wilfrid Laurier University in Waterloo, Canada. In this database, participants in the Parkinson's disease FOG group were selected based on their self-report of experiencing freezing. Initially, patients would be interviewed by a trained clinician (about their experience of freezing) but only if they had scored a 1 or higher on question 14 of the Unified Parkinson's Disease Rating Scale (UPRS). This question specifically addresses whether or not freezing was experienced by the participant at the time they enrolled as a research centre participant. Additionally, a trained clinician confirmed the occurrence of freezing in these patients at the time of the test (see the Procedures subsection below).

All patients who were tested had clinically typical Parkinson's disease as confirmed by diagnosis from at least one movement disorder neurologist and were known to be responsive to antiparkinsonian medication. All participants with Parkinson's disease were tested approximately $1 \mathrm{~h}$ after having taken their anti-Parkinson's medication. However, criteria were used to verify that individuals in the FOG group were experiencing episodes of freezing at the time of the test (see the Procedures subsection below). Participants in the non-FOG Parkinson's disease group scored at least a 1 (out of 4) on the gait portion of the UPDRS-Motor section III by a movement disorder specialist and had no self-reported incidents of freezing in their case history. Sixteen healthy control individuals also participated in the study. These individuals were mostly spouses or relatives of the participants with Parkinson's disease.

Participants were excluded from testing if they had a history of neurological conditions other than Parkinson's disease, or orthopaedic or visual disturbances that severely impaired walking ability. Participants were also excluded if they had been previously diagnosed by a neurologist with dementia, had a confirmed score of $<27$ on the Mini Mental State Examination, or had dyskinesias, which would alter their gait pattern. Each participant was informed about the requirements of the study and signed institutionally approved consents, according to the declaration of Helsinki (BMJ 1991; 302: 1194).

\section{Materials}

The room used for data collection was a laboratory containing a metal-framed double doorway leading into an empty hallway. The double doorway acted as the wide doorway condition (ie, two times normal door width), whereas the single doorway was used for the normal doorway condition. A perfectly colourmatched wooden plank was fitted in to the side of the single doorway for the narrow doorway condition. The lighting in the laboratory and the hallway was maintained at a consistent brightness. Data were collected on a GAITRite carpet (GAITRite, CIR System, Inc., Clifton, New Jersey, USA), which is $3.96 \mathrm{~m}$ long $\times 0.79 \mathrm{~m}$ wide and contains sensors that send information received from the participants' footsteps to an attached computer. A researcher walked alongside (and slightly behind) the participant at all times for safety of the participant during each trial.

\section{Procedure \\ Pretest procedure}

In addition to the UPDRS assessment of gait (used to confirm the presence of freezing), an additional measure was developed to further establish the experience of freezing at the time of the test. Each participant in the Parkinson's disease FOG group performed a modified version (through a doorway) of the timed up-and-go test (TUG). This test began with the patients seated in a chair and, upon a go signal, they proceeded to stand and walk through a doorway to a marker on the ground located $3 \mathrm{~m}$ from the chair. Once they reached this marker, they turned and proceeded back to the chair to sit down. This was observed by a movement disorder specialist who confirmed that the participant was experiencing a sudden inability to initiate or continue walking at some point during the TUG before continuing to the normal testing procedure. If FOG indicators were absent, the patient was excluded from testing

\section{Test procedure}

Participants walked the length of the GAITRite carpet that was positioned so that participants would walk through a doorway, in three randomised conditions that were five (blocked) trials each. Each trial commenced with the participant, with eyes closed, standing $2 \mathrm{~m}$ before the start of a GAITRite carpet. This ensured that characteristics of gait initiation were not recorded. Participants were instructed to start walking as soon as they opened their eyes, and walk the length of the GAITRite carpet through the doorway. The three experimental conditions were:

1. Narrow doorway condition in which the participant walked through a smaller-than-normal (3/4 size) doorway $(0.675 \mathrm{~m}$ wide $\times 2.1 \mathrm{~m}$ high). The wooden plank designed to make the doorway narrow partially overlapped the GAITRite carpet in this condition.

2. The normal doorway condition acted as the baseline control condition in which the participant walked through a normalsized doorway ( $0.9 \mathrm{~m}$ wide $\times 2.1 \mathrm{~m}$ high).

Table 1 Characteristics of the three groups

\begin{tabular}{|c|c|c|c|c|c|c|}
\hline Group & $\begin{array}{l}\text { Age-M } \\
\text { (yrs) }\end{array}$ & Height $-M(\mathbf{c m})$ & $\begin{array}{l}\text { UPDRS-M } \\
\text { (score) }\end{array}$ & $\begin{array}{l}\text { Years since } \\
\text { diagnosis }-M \text { (yrs) }\end{array}$ & Dose of levodopa-M (mg) & Sex \\
\hline Parkinson's disease FOG & $72.4(6.79)$ & $172.51(8.51)$ & $32.8(7.34)$ & $9.07(5.29)$ & $1013.33(390.27)$ & 13 male, 2 female \\
\hline Parkinson's disease Non-FOG & $72.19(6.23)$ & $170.66(9.69)$ & $28.81(6.35)$ & $5.97(4.61)$ & $725.0(449.81)$ & 10 male, 6 female \\
\hline
\end{tabular}


3. Wide doorway condition in which the participant walked through a double-sized doorway (1.8 $\mathrm{m}$ wide $\times 2.1 \mathrm{~m}$ high).

The three conditions in this protocol allowed for the analysis of whether the size of doorway is a contributing factor to the gait alterations and FOG experienced while travelling through confined spaces. Only data of the spatiotemporal gait characteristics collected prior to the doorway were included for analysis. This allowed for the analysis of anywhere from four to seven steps depending on the participants' step length. Any foot falls at or after the doorway were excluded from analysis in this experiment.

\section{Statistical analysis}

There were three independent groups in this experiment: individuals with Parkinson's disease experiencing FOG (Parkinson's disease FOG), those with Parkinson's disease experiencing gait abnormalities absent of FOG (Parkinson's disease non-FOG) and healthy control individuals (Controls). As suggested by Morris et $a l,{ }^{27}$ individuals with Parkinson's disease are known to have deficits in velocity, step length, step timing and base of support. As such, the primary dependent variables analysed were gait velocity $(\mathrm{cm} / \mathrm{s})$, mean step length $(\mathrm{cm})$, which is equal to the length of a toe off to the opposite foot heel contact, base of support $(\mathrm{cm})$, cadence (steps/min), time spent in double support (s). In addition, Hausdorff et al, ${ }^{10}$ have supported the evaluation of step-to-step variability as a precursor of FOG. Hence, two measures of step-to-step variability were calculated for each of the spatiotemporal measures: (1) within-trial SD around each individual participant's mean value for a trial was averaged across participants in a given group, and (2) the coefficient of variation $(\mathrm{CV})$ within a trial was calculated based on SD (see (1)) divided by the average value for a given trial in order to account for variability normalised to the mean. Left and right steps were pooled and the results were analysed by the STATISTICA computerised statistical package using a mixed model of three groups $\times$ three conditions $\times$ five trials analysis of variance (ANOVA). In order to determine where the significant differences found in the ANOVAs occurred, Tukey's Honest Significant Difference (HSD) post hoc procedure was employed.

\section{Role of the funding source}

The funding source behind this research did not have any involvement in the study design; in the collection, analysis and interpretation of data; in the writing of the report; nor in the decision to submit the paper for publication.

\section{RESULTS}

\section{Participant demographics}

To determine whether significant differences existed between the three groups, an ANOVA was performed and found no significant differences between the groups age and height. A t test was also performed and found no significant differences between the Parkinson's disease FOG and Parkinson's disease non-FOG group in regards to their years since diagnosis or the amount of levodopa they were taking. There was also no significant difference found with regards to disease progression as indicated by their UDPRS score.

\section{Gait velocity}

Individuals who experience FOG were found to walk significantly slower $(85.5(30.0) \mathrm{cm} / \mathrm{s})$ on average as compared with the Parkinson's disease non-FOG group $(107.3(18.8) \mathrm{cm} / \mathrm{s})$ and the Control group $(119.3(12.81) \mathrm{cm} / \mathrm{s})$, as demonstrated by a main effect of group $\left(\mathrm{F}_{(2,44)}=10.90, \mathrm{p}<0.001\right)$. Post hoc analysis confirmed that the Parkinson's disease FOG group walked at a significantly slower velocity as compared with the Parkinson's disease non-FOG group $(20.3 \%$ decrease, $p=0.012)$ and the Control group $(28.3 \%$ decrease, $p<0.001)$. There was no significant interaction of velocity observed with condition. An interaction was identified between group and trial $\left(F_{(8,176)}=2.36, p<0.0195\right)$. This interaction demonstrated that the Parkinson's disease FOG group was the only group to experience a reduced velocity in their first encounter with the doorway. Neither the Parkinson's disease non-FOG group nor the Healthy Control group altered their velocity through the trials.

\section{Step length}

It was also observed that the Parkinson's disease FOG group had a significantly smaller step length $(45.9(13.9) \mathrm{cm})$ as compared with the Parkinson's disease non-FOG group $(56.6(7.4) \mathrm{cm})$ and the Control group $(63.9(6.8) \mathrm{cm})$. This was evident from the observed main effect of the group $\left(\mathrm{F}_{(2,44)}=13.11, \mathrm{p}<0.001\right)$. Post hoc analysis confirmed that the Parkinson's disease FOG group had a significantly smaller step length than the Parkinson's disease non-FOG group $(p<0.011)$ and the Control group $(p<0.001)$. The Parkinson's disease non-FOG group did not differ significantly from the Control group.

More importantly, a significant interaction of step length was found when comparing group and condition $\left(\mathrm{F}_{(4,88)}=2.73\right.$, $\mathrm{p}<0.034$ ) (figure 1). The narrow doorway significantly decreased the step length of the Parkinson's disease FOG group, while the other two groups were not affected. This was confirmed through post hoc analysis in which the narrow doorway caused the Parkinson's disease FOG group to shorten their steps (42.5 $(15.4) \mathrm{cm}$ ) as compared with the normal doorway (46.4 $(13.9) \mathrm{cm})$ by $8.4 \% \quad(\mathrm{p}<0.005)$ and wide doorway $(48.7$ $(13.5) \mathrm{cm})$ by $12.7 \%(\mathrm{p}<0.001)$.

An interaction was identified between the group and the trial when examining step length $\left(F_{(8,176)}=6.08, p<0.001\right)$. The Parkinson's disease FOG group demonstrated a significantly smaller step length in their first encounter with the doorway $(43.4(15.5) \mathrm{cm})$ as compared with the other trials $(\mathrm{p}<0.029)$. Neither the Parkinson's disease non-FOG nor the Control group altered their step length with respect to trial.

\section{Base of support}

A significant interaction of group and condition was found when analysing base of support $\left(\mathrm{F}_{(4,88)}=3.96, \mathrm{p}<0.053\right)$ (figure 2). Base of support did not significantly change with

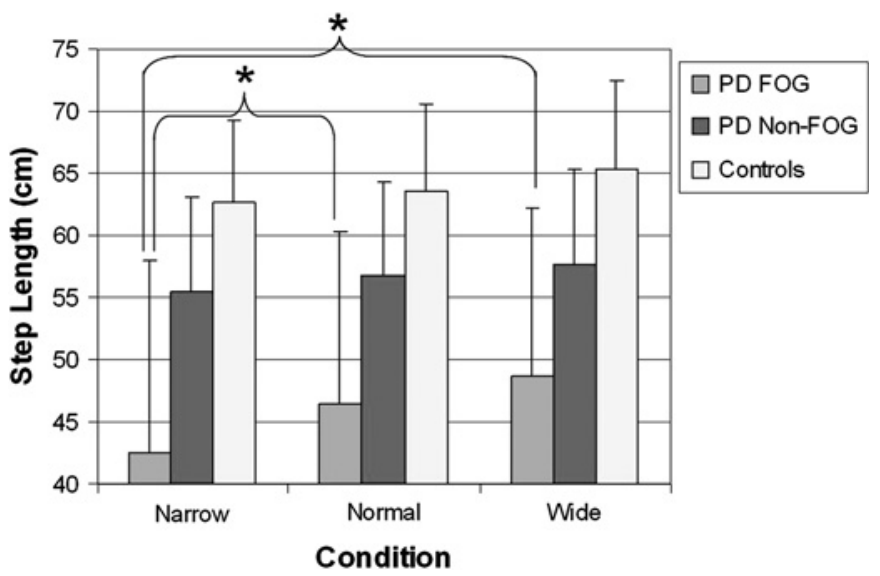

Figure 1 Changes in step length over the three conditions in the Parkinson's disease FOG group, Parkinson's disease non-FOG group and controls. 


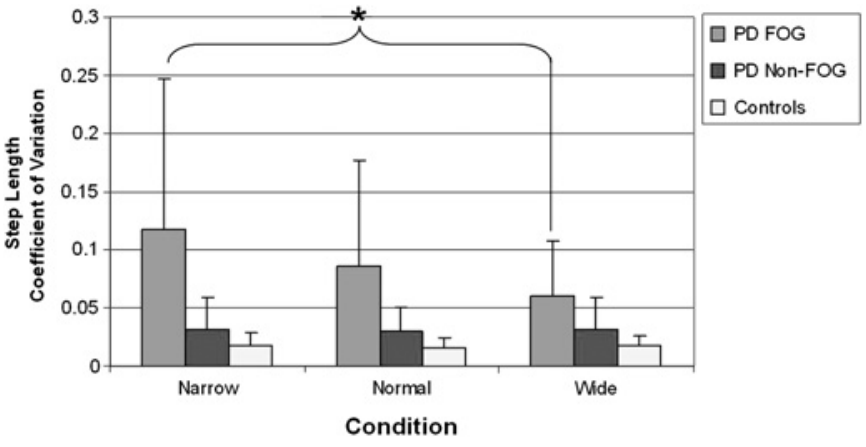

Figure 2 Base of support alterations across condition.

condition in either the Parkinson's disease FOG group or the Control group. Also, it was found that the Parkinson's disease FOG group had a significantly larger base of support (on average, $29.6 \%$ larger) as compared with that of the Control group $(p<0.001)$ across all conditions. The Parkinson's disease FOG groups' base of support was found to be consistently the widest and the Control group the narrowest. In the wide doorway condition, individuals in the Parkinson's disease non-FOG group were found to behave like those of the Control group, with both groups revealing a significantly smaller base of support when compared with the Parkinson's disease FOG group $(p<0.001)$. In the normal doorway, the Parkinson's disease non-FOG group differed from both groups, with a significantly smaller base of support than that of the Parkinson's disease FOG group ( $p<$ $0.001)$ and a wider base of support than that of the Control group $(p<0.004)$. Interestingly, when confronted with the narrow doorway condition, the Parkinson's disease non-FOG group behaved similar to the Parkinson's disease FOG group and only differed from the Controls, who continued to display the narrowest base of support $(p<0.001)$. Solely, the Parkinson's disease non-FOG group altered the size of their base of support with respect to doorway as they exhibited a wider base of support when approaching the narrow doorway $(19.6(6.9) \mathrm{cm})$ as compared with the wide doorway (17.3 (7.7) $\mathrm{cm}, \mathrm{p}<0.035)$.

\section{Step length variability}

When comparing groups for within-trial step length variability, a main effect was found $\left(\mathrm{F}_{(2,44)}=7.79, \mathrm{p}<0.002\right)$. Post hoc analysis confirmed that the Parkinson's disease FOG group (2.9 $(1.5) \mathrm{cm}$ ) had significantly greater step length variability as compared with the Parkinson's disease non-FOG group (1.6 $(0.9) \mathrm{cm}, \mathrm{p}<0.004)$ and the Controls $(1.0(0.5) \mathrm{cm}, \mathrm{p}<0.001)$. A significant interaction was also identified between group and condition $\left(\mathrm{F}_{(4,88)}=2.99, \mathrm{p}<0.023\right)$ (figure 3$)$. The Parkinson's disease FOG group was the only group found to exhibit an increased step length variability in the narrow $(3.2(1.8) \mathrm{cm}$, $\mathrm{p}<0.001)$ doorway and the normal $(3.0(1.8) \mathrm{cm}, \mathrm{p}<0.091)$ doorway as compared to the wide $(2.4(1.2) \mathrm{cm}$,) doorway condition. Neither the Parkinson's disease non-FOG group nor the Control group demonstrated a change in step length variability as a result of doorway condition.

In order to normalise against mean values, the CV of step length was analysed, revealing a significant group versus condition interaction $\left(\mathrm{F}_{(4,88)}=2.85, \mathrm{p}<0.029\right)$ (figure 4). Solely, the Parkinson's disease FOG group was affected by the size of doorway, as they experienced a higher CV with regards to step length in the narrow doorway $(0.118,(0.129))$ as compared with the wide doorway $(0.06(0.048), \mathrm{p}<0.004)$.

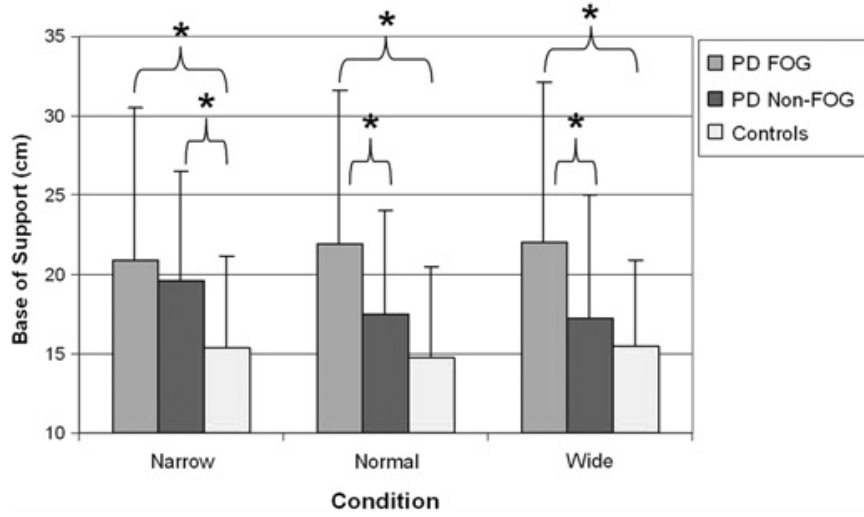

Figure 3 Parkinson's disease FOG group displays increased step length variability in narrow condition.

\section{Step duration}

Without the data being normalised, there were no significant group, condition or trial effects with regards to step duration. However, the CV of step duration was analysed and revealed a main effect of group. The Parkinson's disease FOG group was found to have a significantly higher CV $(0.085(0.088))$ as compared with the Parkinson's disease non-FOG group $(0.031$ (0.02), $\mathrm{p}<0.015)$ and the Control group $(0.018$ (0.007), $\mathrm{p}<0.002)$ groups $\left(\mathrm{F}_{(2,44)}=7.45, \mathrm{p}<0.002\right)$. Also, a trend reaching significance was found between group and condition, indicating that the participants in the Parkinson's disease FOG group were the only ones affected by doorway size (with respect to CV of step duration).

\section{DISCUSSION}

The primary objective of the current study was to evaluate the influence of space perception on gait in individuals with Parkinson's disease who experience FOG, other Parkinson's disease patients (absent of FOG) and healthy age-matched participants. Freezing is extremely difficult to draw out in experimental settings, ${ }^{28}$ as was also the case during the current experiment, which has been suggested to be caused by a heightened attention due to participation in an experiment. ${ }^{9}$ However, several studies have shown decreased stride length and increased gait timing variability before a freezing episode. ${ }^{10}$ Therefore, in spite of a lack of actual freezing episodes, the obtained results demonstrate that an upcoming confined space has a profound effect on gait in patients experiencing FOG. Overall, the gait of the FOG participants was significantly more variable when compared with the

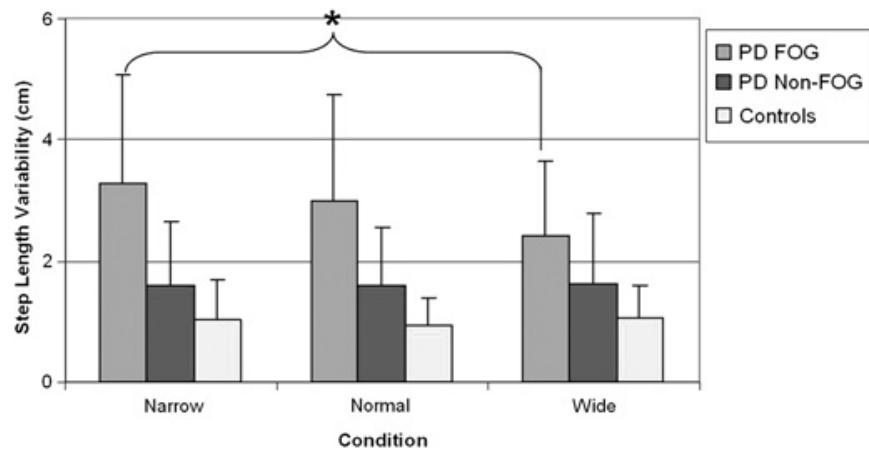

Figure 4 Step length coefficient of variation over the three conditions in the Parkinson's disease FOG, Parkinson's disease non-FOG and control groups. 
other groups (as demonstrated by CV data for step length and step duration), which is in agreement with previous research demonstrating an increase in gait variability before a freeze. ${ }^{10}$ Perhaps important to note is that these indicators of freezing are occurring well before arrival at the actual doorway, suggesting that online perceptual processes must be interrupting the initial movement plan to pass through the doorway. Thus, impaired perceptual ability may be an important factor contributing to freezing in Parkinson's disease.

This is the first study to demonstrate that while approaching a narrow doorway, freezers already exhibit alterations to gait (shortened step length, increased gait variability, increased base of support) that are indicative of an upcoming freezing episode. These changes were not evident in non-FOG individuals with Parkinson's disease, or healthy participants. In fact, non-FOG individuals showed a constant deficit in step length (compared with healthy ones), regardless of doorway size. Our results are in direct contrast to Van Wegen et al who demonstrated that small spaces presented in the form of a virtual corridor had no effect on gait in Parkinson's disease. ${ }^{28}$ A group by trial interaction reaffirms that the Parkinson's disease FOG group were most affected (in terms of step length and velocity) upon their first encounter with the doorway, whereas the two other groups were unaffected by trial. This may suggest that experience (ie, practice) helps Parkinson's disease patients improve their spatial perception to confirm a door size, although heightened anxiety level (in a group of patients that are prone to falling) during the first encounter with the narrow cannot be ruled out. Therefore, perceptual judgment of the upcoming doorway, and thus a certain degree of visuospatial ability, appears to be more greatly affected in Parkinson's disease patients who experience freezing.

Base of support is generally considered to be a measure of stability, and hence, we hypothesised that the Parkinson's disease FOG group might attempt to maximise stability by increasing base of support in the narrow doorway condition. However, the Parkinson's disease FOG group showed a consistent increase in base of support that was not significantly influenced by doorway size. Since freezers are substantially more unstable, they may adopt a wide base of support regardless of environmental context. Interestingly, the Parkinson's disease non-FOG group altered their base of support only in anticipation of the narrow doorway (similar to freezers) and yet were not affected by the other doorways. In contrast, healthy control participants have a high level of stability and hence, maintained a normal (and narrower) base of support regardless of condition (compared with the Parkinson's disease groups).

In accordance with Hausdorff et al, ${ }^{10} 29$ the current study found increased within-trial step length and step duration variability in only the FOG group, and this was more profound in the narrow doorway specifically. It should be noted that they were the only group to demonstrate this effect, providing additional support to the hypothesis that perceptual impairments primarily affect individuals with freezing. The increased step length variability is indicative of an unstable gait pattern that may be reflective of an attempt to voluntarily control gait, possibly by increasing the sampling of proprioceptive feedback. ${ }^{16}$ Freezers were unable to maintain a normal stride and instead more frequently altered their step length, potentially leading to an increased risk of falling. ${ }^{30}$ As suggested by Iansek et $a l,{ }^{31}$ the fact that a decreased step length (accompanied by increased variability) can be identified before the narrow doorway suggests that attentional or perceptual mechanisms (ie, involved in processing characteristics of the door) contribute to the occurrence of a freezing episode.
Although perception was not directly evaluated, our work has provided a glimpse of the impact that perceptual mechanisms may have on severe gait deficits such as freezing. Changes in step length, base of support and within-trial step length and step duration variability all support the notion that patients with FOG alter their gait in response to how they perceive environmental contexts. This may be important to consider since the observed changes in gait can be predictive of an upcoming freezing episode. ${ }^{10} 12$ Parkinson's disease patients without FOG were also found to be affected by narrow doorways, suggesting that increased perceptual constraints may lead to gait alterations even in non-freezers. Individuals with Parkinson's disease appear to be unable to accurately evaluate self-motion in relation to upcoming obstacles. ${ }^{22}$ This may be an important perceptual factor to consider for other situations such as entering an elevator or any other situation in which patients may be approaching confined or crowded spaces. We recognise that there are certain situations that elicit FOG (ie, turning) that may not be related to perception. Suggestive mechanisms for freezing during turning include asymmetrical gait. $^{32}$

As previously mentioned, freezing is extremely difficult to draw out in laboratory settings; thus, it is important to consider whether patients categorised as non-freezers may have had the experience of FOG within their own home environment. In the case of the current study, categorisation into the non-FOG group was confirmed by a movement disorders trained clinician, in addition to self-report (of experience at home), UPDRS (Q. 14) and our modified TUG test. While the possibility exists that the non-FOG patients might progress into FOG, it is unlikely that they would be experiencing any sort of FOG at the present time.

Individuals with Parkinson's disease were tested while on dopaminergic medication, which is a potential limitation of this study, although it is has been shown that freezing is poorly affected by medication. ${ }^{17}$ Testing was conducted solely in the "on" state of Parkinson's disease in order to get a true understanding of the perceptual mechanisms that may commonly occur while patients are medicated (as in everyday situations). Future studies might include the testing of individuals with FOG while "off" medication in order to obtain a clearer understanding of basal ganglia contribution to freezing. The use of an eye tracking device in future research could be useful in order to monitor participants gaze directions while approaching the doorway. Future research also should focus on underlying perceptual mechanisms that may be prevalent in FOG (and more generally in Parkinson's disease) in order to better understand the causes of freezing. Taking these perceptual mechanisms into consideration will be important for the development of effective treatment strategies to combat freezing.

Acknowledgements This study was supported by the Natural Sciences and Engineering Research Council of Canada (NSERC).

Funding The Natural Sciences and Engineering Research Council of Canada (NSERC)

Competing interests The authors state that there are no conflicts of interest with regards to this research.

Ethics approval The research and ethics board at Wilfrid Laurier University in Waterloo, Ontario, Canada approved this study.

Patient consent Obtained.

Provenance and peer review Not commissioned; externally peer reviewed.

\section{REFERENCES}

1. Bloem BR, Grimbergen YA, Cramer M, et al. Prospective assessment of falls in Parkinson's disease. J Neurol 2001;248:950-8. 
2. Moore 0, Peretz C, Giladi N. Freezing of gait affects quality of life of peoples with Parkinson's disease beyond its relationships with mobility and gait. Mov Disord 2007;22:292-5.

3. Bloem BR, Hausdorff JM, Visser JE, et al. Falls and freezing of gait in Parkinson's disease: a review of two interconnected, episodic phenomena. Mov Disord 2004;19:871-84.

4. Giladi N, McMahon D, Przedborski S, et al. Motor blocks in Parkinson's disease. Neurology 1992;42:333-9.

5. Bartels AL, Balash Y, Gurevich $T$, et al. Relationship between freezing of gait (FOG) and other features of Parkinson's: FOG is not correlated with bradykinesia. J Clin Neurosci 2003;10:584-8.

6. Okuma Y. Freezing of gait in Parkinson's disease. J Neuro/ 2006;253(Suppl 7):VII27-32

7. Schaafsma JD, Balash $Y$, Gurevich $T$, et al. Characterization of freezing of gait subtypes and the response of each to levodopa in Parkinson's disease. Eur $\mathrm{J}$ Neurol 2003:10:391-8.

8. Amboni M, Cozzolino A, Longo K, et al. Freezing of gait and executive functions in patients with Parkinson's disease. Mov Disord 2008:23:395-400.

9. Nieuwboer $\mathbf{A}$, Giladi N. The challenge of evaluating freezing of gait in patients with Parkinson's disease. Br J Neurosurg 2008;22(Suppl 1):S16-8.

10. Hausdorff JM, Schaafsma JD, Balash Y, et al. Impaired regulation of stride variability in Parkinson's disease subjects with freezing of gait. Exp Brain Res 2003;149:187-94.

11. Nieuwboer A, Dom R, De Weerdt W, et al. Electromyographic profiles of gait prior to onset of freezing episodes in patients with Parkinson's disease. Brain 2004;127 (Pt 7):1650-60.

12. Nieuwboer A, Dom R, De Weerdt W, et al. Abnormalities of the spatiotemporal characteristics of gait at the onset of freezing in Parkinson's disease. Mov Disord 2001:16:1066-75

13. Nieuwboer A, De Weerdt W, Dom R, et al. Plantar force distribution in Parkinsonian gait: a comparison between patients and age-matched control subjects. Scand J Rehabil Med 1999:31:185-92.

14. Morris ME. Locomotor training in people with Parkinson disease. Phys Ther 2006:86:1426-35.

15. Almeida 0J, Wishart LR, Lee TD. Bimanual coordination deficits with Parkinson's disease: the influence of movement speed and external cueing. Mov Disord 2002;17:30-7

16. Almeida $\mathbf{Q J}$, Frank JS, Roy EA, et al. Dopaminergic modulation of timing control and variability in the gait of Parkinson's disease. Mov Disord 2007;22:1735-42.
17. Giladi N, Huber-Mahlin V, Herman T, et al. Freezing of gait in older adults with high level gait disorders: association with impaired executive function. J Neural Transm 2007:114:1349-53.

18. Factor SA, Jennings DL, Molho ES, et al. The natural history of the syndrome of primary progressive freezing gait. Arch Neurol 2002;59:1778-83.

19. Lieberman A. Are freezing of gait (FOG) and panic related? J Neurol Sci 2006;248:219-22.

20. Giladi N, Hausdorff JM. The role of mental function in the pathogenesis of freezing of gait in Parkinson's disease. J Neurol Sci 2006;248:173-6.

21. Bodis-Wollner I. Neuropsychological and perceptual defects in Parkinson's disease. Parkinsonism Relat Disord 2003;9(Suppl 2):S83-9.

22. Almeida 0J, Frank JS, Roy EA, et al. An evaluation of sensorimotor integration during locomotion toward a target in Parkinson's disease. Neuroscience 2005; 134:283-93.

23. Gurvich C, Georgiou-Karistianis N, Fitzgerald PB, et al. Inhibitory control and spatial working memory in Parkinson's disease. Mov Disord 2007;22:1444-50.

24. Johnson AM, Almeida OJ, Stough C et al. Visual inspection time in Parkinson's disease: deficits in early stages of cognitive processing. Neuropsychologia 2004:42:577-83

25. Davidsdottir S, Cronin-Golomb A, Lee A. Visual and spatial symptoms in Parkinson's disease. Vision Res 2005;45:1285-96.

26. Lee AC, Harris JP. Problems with perception of space in Parkinson's disease. NeuroOphthalmology 1999:22:1-15.

27. Morris ME, lansek R, Matyas TA, et al. The pathogenesis of gait hypokinesia in Parkinson's disease. Brain 1994;117(Pt 5):1169-81.

28. van Wegen $\mathbf{E}$, Lim I, de Goede C, et al. The effects of visual rhythms and optic flow on stride patterns of patients with Parkinson's disease. Parkinsonism Relat Disord 2006:12:21-7

29. Hausdorff JM, Cudkowicz ME, Firtion R, et al. Gait variability and basal ganglia disorders: stride-to-stride variations of gait cycle timing in Parkinson's disease and Huntington's disease. Mov Disord 1998;13:428-37.

30. Schaafsma JD, Giladi N, Balash Y, et al. Gait dynamics in Parkinson's disease: relationship to Parkinsonian features, falls and response to levodopa. J Neurol Sci 2003;212:47-53.

31. lansek R, Huxham F, McGinley J. The sequence effect and gait festination in Parkinson disease: contributors to freezing of gait? Mov Disord 2006:21:1419-24.

32. Plotnik M, Giladi N, Balash $Y$, et al. Is freezing of gait in Parkinson's disease related to asymmetric motor function? Ann Neurol 2005;57:656-63. 